\title{
Treatment of glaucomatous optic nerve damage using ginsenoside Rg1 mediated by ultrasound targeted microbubble destruction
}

\author{
LIANFENG WANG, TINGTING CAO and HAITING CHEN \\ Section One, Department of Ophthalmology, Cangzhou Central Hospital, Cangzhou, Hebei 061001, P.R. China
}

Received June 21, 2017; Accepted October 10, 2017

DOI: $10.3892 / \mathrm{etm} .2017 .5386$

\begin{abstract}
The treatment of glaucomatous optic nervedamage using ginsenoside Rg1 mediated by ultrasound targeted microbubbles destruction was evaluated. Thirty healthy New Zealand white rabbits were subjected to injection of $0.3 \%$ carbomer solution to establish glaucomatous optic nerve damage model. Rabbits were divided into 5 groups: control group, model group, model group + intravitreal injection of nerve growth factor (NGF) group, model group + intravitreal injection of ginsenoside $\mathrm{Rg} 1$ group ( $\mathrm{Rg} 1$ group), model group + intravitreal injection of ginsenoside Rg1 + ultrasound microbubble group (ultrasound group), model group + ultrasound targeted microbubble destruction (ultrasound group). Intraocular pressures were compared at 1,2 and 4 weeks after model establishment. Rabbits were sacrificed 4 weeks after model establishment to collect retinal tissue for H\&E staining. Histological changes were observed and the retinal thickness was measured. Contents of malondialdehyde (MDA), superoxide dismutase (SOD) and nitric oxide (NO) were measured by ELISA. Intraocular pressure was significantly higher in model group than in control group at 1 week $(\mathrm{P}<0.05)$. Intraocular pressure was significantly lower in the ultrasound group than in NGF group and Rg1 group at all time-points $(\mathrm{P}<0.05)$. The number of ganglion cells in model group was decreased significantly. Number of nuclear layer cells was significantly reduced. Thickest retina was found in control group and model group was the thinnest $(\mathrm{P}<0.05)$. Contents of MDA and NO in model group were significantly higher than those in NCF group and Rg1 group. SOD content in control group was higher than that in ultrasound group and model group $(\mathrm{P}<0.05)$. In conclusion, treatment of glaucomatous optic nerve damage using ginsenoside Rg1 mediated by ultrasound targeted microbubble destruction can reduce the level
\end{abstract}

Correspondence to: Dr Lianfeng Wang, Section One, Department of Ophthalmology, Cangzhou Central Hospital, 16 Xinhuaxi Road, Cangzhou, Hebei 061001, P.R. China

E-mail:wma2sq@163.com

Key words: ultrasound targeted microbubble destruction, ginsenoside Rg1, nerve growth factor, glaucoma, malondialdehyde, superoxide dismutase, nitric oxide of oxidative stress, relieve intraocular pressure and reduce ganglion cell damage.

\section{Introduction}

Glaucoma is the second most common eye disease leading to blindness in the world with high intraocular pressure, irreversible optic atrophy and visual field defects as the main features. Clinical treatment mainly aims to reduce intraocular pressure and maintain the normal range of intraocular pressure, main treatments include laser, drugs, surgery and other methods (1). Basic study confirmed that (2) retinal ganglion cell (RGC) apoptosis and optic nerve axon degeneration caused by ischemia, oxidative stress and inflammatory response are important causes of the occurrence and development of glaucoma. Malondialdehyde (MDA), nitric oxide (NO) and superoxide dismutase (SOD) are the most commonly used biochemical markers for the evaluation of glaucoma animal model and glaucoma patients with oxidative stress disorder. The use of optic nerve protection drugs such as nerve growth factor (NGF) can prevent or delay the damage of RGCs, so as to improve glaucoma symptoms and prognosis (3). Drug application paths mainly include oral medication, and intravenous and intramuscular injection, the drug reaches the eyes through circulation system, which in turn lead to the relative low effective concentrations of drugs in area around retina and optic nerve, leading to the low efficacy, at the same time, the incidence of toxic side effects will be increased (4). Therefore, the development of a more efficient and safe way of drug administration will definitely improve the clinical treatment of glaucoma. Drug release mediated by ultrasound targeted microbubble destruction can achieve accurate positioning and targeted release, which in turn reduces the dose, improves clinical results, and reduces systemic adverse reactions (5). Ginsenoside $\mathrm{Rg} 1$ has proven anti-fatigue, antiaging, anticancer, lipid-lowering, memory enhancement, immunity improvement and other pharmacological effects (6). Based on this, we investigated the mechanism of treatment of glaucomatous optic nerve damage using ginsenoside Rg1 mediated by ultrasound targeted microbubble destruction.

\section{Materials and methods}

Experimental materials. Thirty healthy New Zealand white rabbits $(2-2.5 \mathrm{~kg})$ without restriction on sex were purchased 
from Sangon (Shanghai, China). Rabbits were raised under normal conditions for 1 week to be familiar with the environment before the experiment. The study was approved by the Ethics Committee of Cangzhou Central Hospital.

Establishment of glaucomatous optic nerve damage model: Intramuscular injection of sumianxin II $(0.2 \mathrm{ml} / \mathrm{kg})$ for anesthesia, dicaine hydrochloride eye drops was used for topical anesthesia; aqueous humor $(0.2 \mathrm{ml})$ was extracted from anterior chamber along one side of corneoscleral in the direction of 9 o'clock, $0.2 \mathrm{ml}$ of compound carbomer solution $(0.3 \%)$ was injected into the anterior chamber along the other side of corneoscleral; ofloxacin eye drops was used. Intraocular pressure measured by accumen hand-held tonometer $>22 \mathrm{~mm}$ $\mathrm{Hg}$ for 4 weeks indicate the successfully established model; if intraocular pressure $<22 \mathrm{mmHg}$, drug administration can be repeated 7 days later.

Main reagent: Ginsenoside Rg1 powder (purity $\geq 98 \%$ ) was purchased from Beyotime Biotechnology (Jiangsu, China), NGF injection was purchased from Beijing Zhongshan Golden Bridge Biotechnology Co., Ltd. (Beijing, China). Sulfur hexafluoride microbubbles for injection was purchased from Bracco Inc. (Milan, Italy), medical ultrasonic coupling agent was from Sigma (St. Louis, MO, USA), MDA, SOD and NO, enzyme-linked immunosorbent assay (ELISA) kits were purchased from R\&D (Minneapolis, MN, USA).

Main instruments: ophthalmic microsurgical instruments were purchased from Beijing Liuyi Instrument Factory (Beijing, China), pathology slicing machine was from Leica (Wetzlar, Germany), optical microscope was purchased from Olympus (Tokyo, Japan), Accupen handheld tonometer was from Accutome Inc. (Malvern, PA, USA).

Research methods. Rabbits were divided into 5 groups: control group, model group, model group + intravitreal injection of nerve growth factor NGF group (NGF group), model group + intravitreal injection of ginsenoside $\mathrm{Rg} 1$ group $(\mathrm{Rg} 1$ group), model group + intravitreal injection of ginsenoside Rg1 + ultrasound microbubble group (ultrasound group), model group + ultrasound targeted microbubble destruction (ultrasound group), 6 rabbits in each group. Methods of intravitreal injection: compound tropicamide eye drops were used for mydriasis, eyelid was opened with eye speculum, conjunctival sac was rinsed with $3 \%$ iodophor. One milliliter syringe was injected vertically into vitreous cavity through the position $3 \mathrm{~mm}$ away from corneoscleral limbus to extract $0.1 \mathrm{ml}$ vitreous humor, then $0.1 \mathrm{ml}$ ginsenoside $\mathrm{Rg} 1$ solution $(2.5 \mathrm{mg} / \mathrm{kg})$ or NGF solution $(18 \mu \mathrm{g} / 0.1 \mathrm{ml})$ was injected, once per 3 days for 4 weeks. Methods of ultrasound targeted microbubbles destruction: After the injection of ginsenoside $\operatorname{Rg} 1$ solution or NGF solution, $0.1 \mathrm{ml}$ microbubble suspension $(45 \mu \mathrm{g} / 0.1 \mathrm{ml})$ and coupling agent coating was performed after the eyes were closed. Ultrasonic probe was places above the eyes to irradiate the eyes for $60 \mathrm{sec}$, once per 3 days for 4 weeks.

Observation indicators. Intraocular pressures were compared at 1,2 and 4 weeks after model establishment. Rabbits were sacrificed 4 weeks after model establishment to collect retinal tissue for H\&E staining. Histological changes were observed and the retinal thickness was measured under a light microscope. Contents of MDA, SOD and NO were measured by
ELISA. All operations were performed in strict accordance with the instructions of kits.

Statistical analysis. Statistical analysis was performed using SPSS 20.0 software (SPSS Inc., Chicago, IL, USA). Measurement data were expressed as mean \pm standard deviation. Single-factor ANOVA analysis was used for the comparisons among multiple groups, and LSD-t method was used for the comparisons between two groups, intraocular pressure within a group was compared with the analysis of variance of repeated measure data. $\mathrm{P}<0.05$ was considered to be statistically significant.

\section{Results}

Comparison of intraocular pressure. Intraocular pressure was significantly higher in the model group than in the control group at 1 week after model establishment, and the peak was reached at 2 weeks after model establishment. Intraocular pressure of NGF group and Rg1 group was significantly lower than that of model group $(\mathrm{P}<0.05)$, but no significant difference was found between NGF group and Rg1 group ( $\mathrm{P}>0.05)$. Intraocular pressure was significantly lower in ultrasound group than in NGF group and Rg1 group at all time-points $(\mathrm{P}<0.05)$, and no significant difference was found between ultrasound group and control group at 4 weeks after model establishment ( $\mathrm{P}>0.05)$ (Fig. 1).

Comparison of histomorphologic structure and retinal thickness. Retinal histomorphology: H\&E staining showed that the RGCs in control group were arranged in a single layer with different sizes, deep nuclear staining, clear boundary and neat arrangement, relative more cells with small nucleus and deep staining were found in outer nuclear layer, cells with neat arrangement, big nucleus and deep staining were found in the inner layer, inner and outer plexus layers showed obvious network structure; in model group, cell vacuolization was common, inner layer of retina was thinned, the nucleolus was dissolved, the nuclei staining was light, color of cytoplasm was light, number of cells in inner and outer nuclear layers was decreased, the gap was widened, cells were arranged sparsely without clear boundaries, inner plexus layers was significantly thinner or even disappeared; number of RGCs in NGF group and Rg1 was reduced but higher than model group, cell vacuolization and karyolysis were rare, cells in inner and outer nuclear layers were arranged sparsely, and inner and outer plexus layers were slightly thinned; there was no decrease in RGCs was found in the ultrasound group, and most of the nuclei were present, disorder in the arrangement of cells in inner and outer nuclear layers was not obvious (Fig. 2).

As shown in Fig. 2A in the control group, cells were arranged in a single layer with different sizes, deep nuclear staining, clear boundary and neat arrangement, relative more cells with small nucleus and deep staining were found in outer nuclear layer, cells with neat arrangement, big nucleus and deep staining were found in inner layer, inner and outer plexus layers showed obvious network structure. In Fig. 2B in the model group, number of RGCs was significantly reduced, cell vacuolization was common, inner layer of retina was thinned, karyolysis occurred, the nuclei staining was light, color of cytoplasm was light, number of cells in inner and 


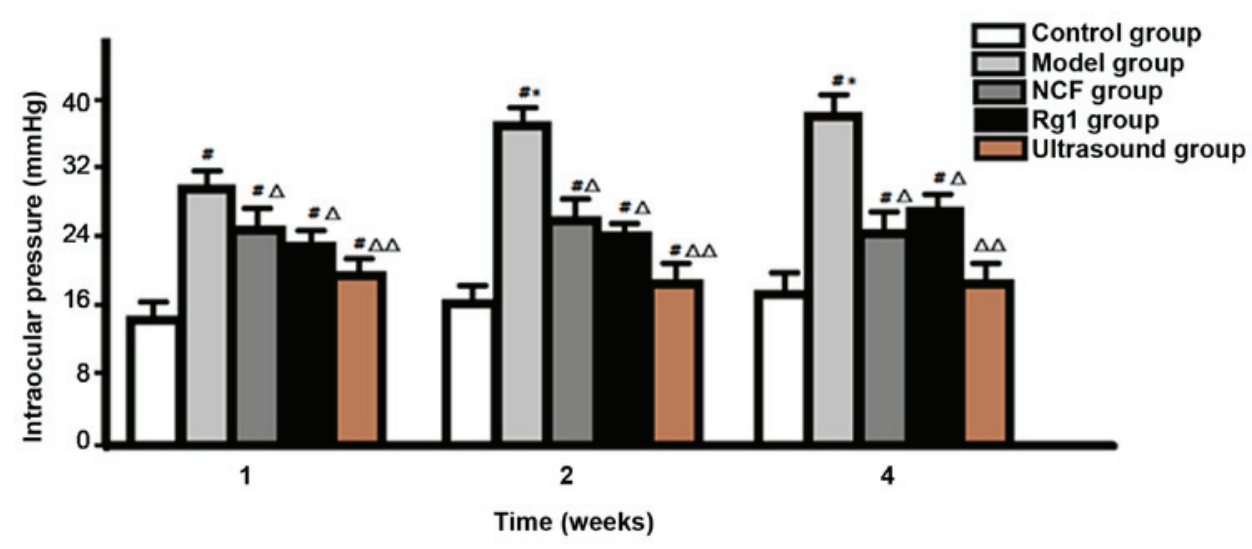

Figure 1. Comparison of intraocular pressure at different time-points after model establishment. ${ }^{\|} \mathrm{P}<0.05$ compared with control group; ${ }^{\wedge} \mathrm{P}<0.05$ compared with control group at the same time-point; ${ }^{\triangle} \mathrm{P}<0.05$ compared with nerve growth factor (NGF) group and $\mathrm{Rg} 1$ group at the same time-point. "P<0.05 compared with NGF group and Rg1 group.

A

B

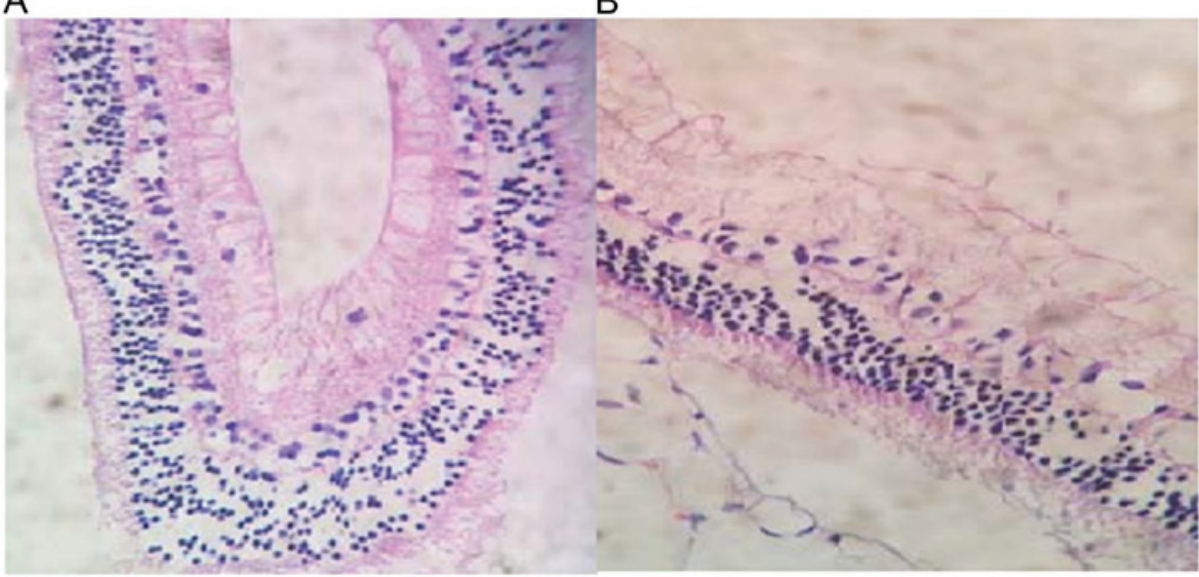

C

D

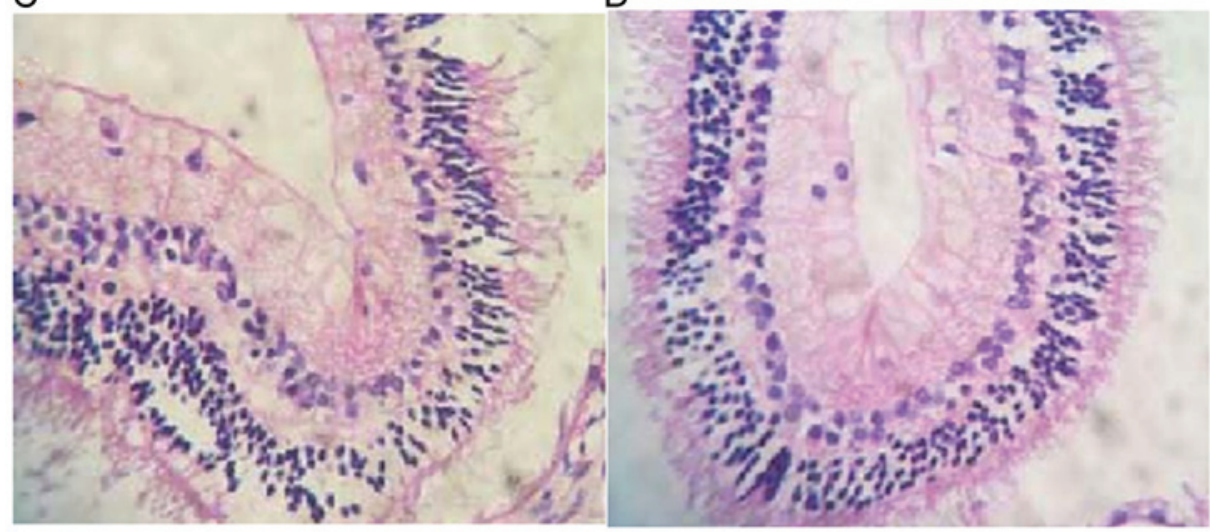

Figure 2. Comparison of histomorphologic structure and retinal thickness at 2 and 4 weeks after model establishment.

outer nuclear layers was decreased, the gap was widened, cells were arranged sparsely without clear boundaries, inner plexus layers was significantly thinner or even disappeared. In Fig. $2 \mathrm{C}$ in the NGF group and $\mathrm{Rg} 1$ group, number of RGCs was reduced but higher than model group, cell vacuolization and karyolysis were rare, cells in inner and outer nuclear layers were arranged sparsely, and inner and outer plexus layers were slightly thinned. No decrease in RGCs was found, most of the nuclei were present, disorder in the arrangement of cells in inner and outer nuclear layers was not obvious (Fig. 2D).
Retinal thickness: The retina in control group was significantly thicker than that in ultrasound group $(\mathrm{P}<0.05)$, followed by NGF group and Rg1 group, the model group was the thinnest (Fig. 3).

Comparison of MDA, SOD and NO contents among groups. Levels of MDA and NO in retina of model group were significantly higher than those in NGF group and Rg1 group, and control group was the lowest. SOD content in control group was higher than that in ultrasound group, followed by NGF 


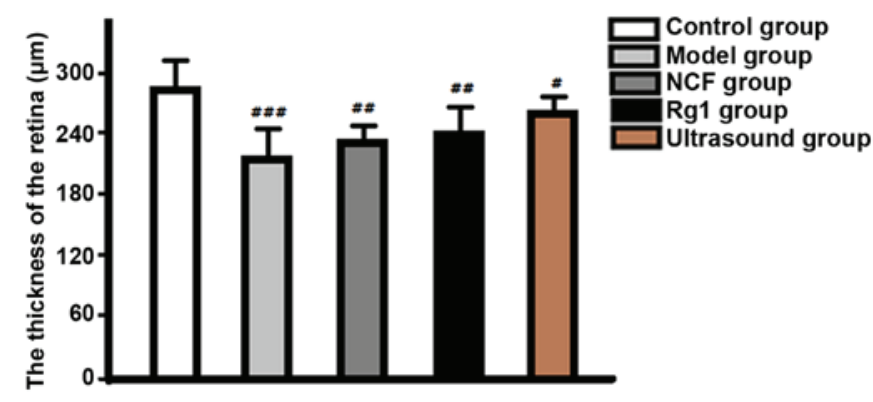

Figure 3. Comparison of the thickness of the retina between groups at 4 weeks after model establishment. Retina in control group was significantly thicker than that in ultrasound group; retina in ultrasound group was significantly thicker than that in nerve growth factor (NGF) group and Rg1 group, retina in NGF group and Rg1 group was significantly thicker than that in model group, the differences were statistically significant $\left({ }^{\#} \mathrm{P}<0.05\right.$ compared with control group; ${ }^{\#} \mathrm{P}<0.05$ compared with ultrasound group; ${ }^{\# \#} \mathrm{P}<0.05$ compared with NGF group and Rg1 group).

group and $\mathrm{Rg} 1$ group, and the differences were all statistically significant $(\mathrm{P}<0.05)$ (Fig. 4).

\section{Discussion}

In this study, the compound carbomer-induced rabbit glaucoma model was used. Results showed that the average intraocular pressure at 1 week after model establishment was $30 \mathrm{mmHg}$, and the peak $(38 \mathrm{mmHg})$ was reached at 2 weeks after model establishment; retinal histomorphological changes were significant and the thickness was significantly decreased, indicating that the intraocular pressure of the rabbit model was significantly increased after carbomer treatment, the duration is long. This method is easy for operation and control, so it is an ideal model for the studies on glaucomatous optic nerve damage and anti-glaucoma drug screening. As the key cytokine involved in optic nerve development, NGF regulates the growth, differentiation and apoptosis of RGCs (7). Different concentrations of NGF added to neonatal rat RGC cell culture medium can promote the survival of RGCs and optic nerve axon elongation, so as to achieve a certain therapeutic effect (8). Recent studies have found that ginsenoside Rg1 can play a neuroprotective by antagonizing dopamine to alleviate the excitotoxicity of neuronal amino acids and reduce neuronal amino acid excitotoxicity, and reduce the toxic effects of phenylfenapyridine $(9,10)$. $\operatorname{Rg} 1$ can upregulate the expression of glial cell derived and brain-derived NGF and other genes (11), promote axonal regeneration, increase the expression of $\mathrm{Bcl}-2$ protein and reduce the expression of Bax protein (12), improve mitochondrial structure and dysfunction, inhibit optic neuronal apoptosis, and block $\mathrm{Ca}^{2+}$ influx (13). Results showed that, compared with model group, NGF and Rg1 can significantly reduce intraocular pressure, improve retinal tissue morphologic structure, increase the thickness of the retina, reduce MDA and NO content, and increase SOD content.

Ultrasound microbubbles achieved great success in the diagnosis and treatment of neoplastic diseases (14), ultrasonic microbubbles were formed by two-part shell and gas inside. After ultrasonic irradiation, drug-containing microbubbles will be destroyed to release drug and cause reversible or irreversible
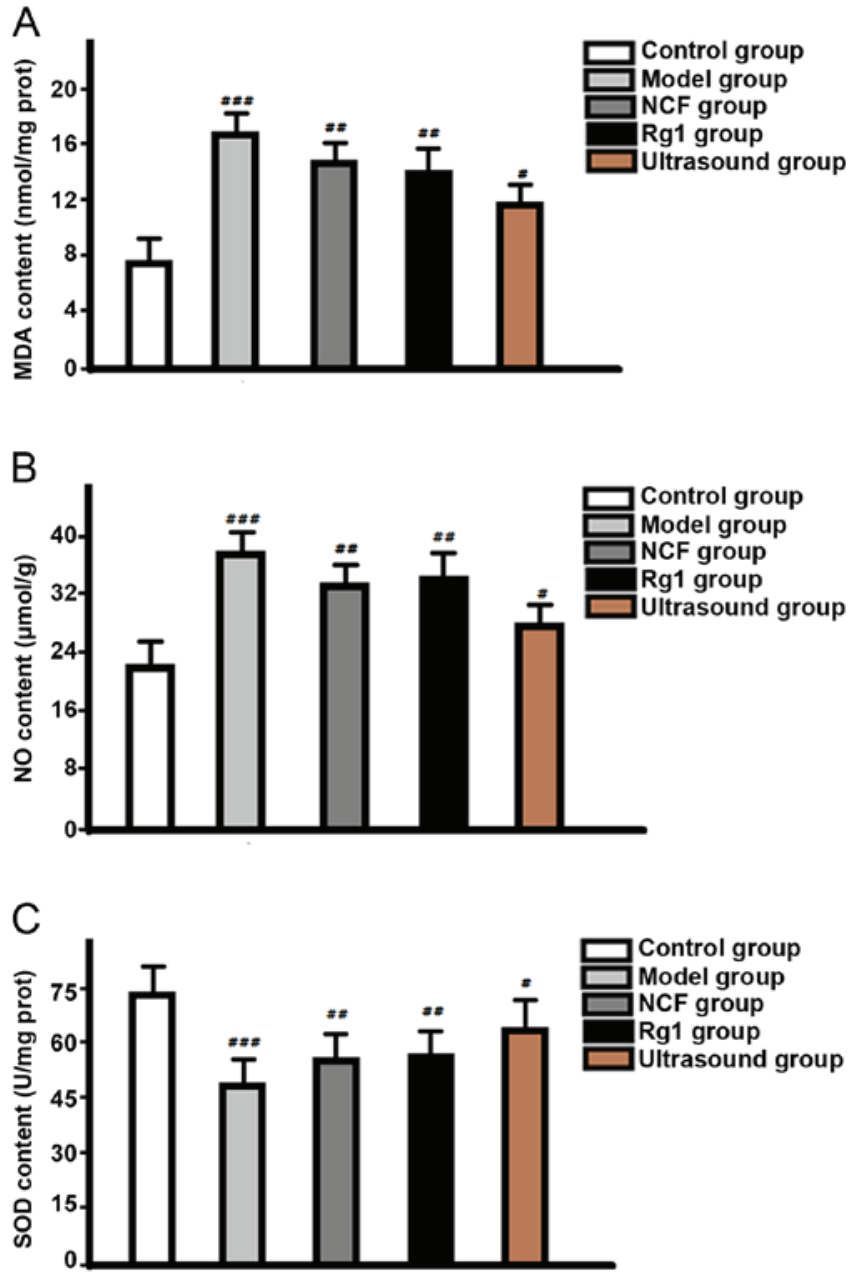

Figure 4. Comparison of MDA, superoxide dismutase (SOD) and nitric oxide (NO) contents in retina after 4 weeks of model establishment. ${ }^{\#}$ Contents of MDA and NO in retina of control were lower than those in ultrasound group, SOD was higher than that in ultrasound group; ${ }^{\#}$ Contents of MDA and NO in ultrasound group were lower than those in nerve growth factor (NGF) group and Rg1 group, SOD content was higher than that in NGF group and Rg1 group; ${ }^{\# \#}$ contents of MDA and NO in NGF group and $\mathrm{Rg} 1$ group were lower than those in model group, SOD level was higher than that in model group, the differences were all statistically significant $(\mathrm{P}<0.05)$.

pores to increase the permeability of the cell membrane, which in turn achieve targeted drug administration $(14,15)$. In the field of ophthalmology, ultrasound microbubbles are mainly used for gene transfection, such as ultrasound microbubble technology to improve the transfection rate DNA plasmid in transfecting retinoblastoma cells (15). Results of this study showed that ultrasound group can further reduce the intraocular pressure, improve retinal histomorphological changes and increase retinal thickness compared with drug treatment alone (NGF group and Rg1 group). In addition, content of MDA and $\mathrm{NO}$ in retinal tissue of ultrasound group was lower than that of NGF group and Rg1 group, and the content of SOD was higher than that of NGF group and Rg1 group, and the differences were statistically significant, indicating that the use of ultrasound microbubbles can achieve targeted drug administration to increase the drug concentration in eyes. So, ginsenoside Rg1 and NGF mediated by ultrasound targeted microbubble destruction is effective in the treatment of glaucomatous optic 
nerve damage, which may be related to the changed contents of NO, MDA and SOD (16-18). This study provided references for the studies on mechanism of the role of oxidative stress injury in the occurrence and development of glaucoma.

\section{References}

1. Quigley HA: Understanding Glaucomatous Optic Neuropathy: The synergy between clinical observation and investigation. Annu Rev Vis Sci 2: 235-254, 2016.

2. Chen X and Zhao Y: Diagnostic performance of isolated-check visual evoked potential versus retinal ganglion cell-inner plexiform layer analysis in early primary open-angle glaucoma. BMC Ophthalmol 17: 77, 2017

3. Oddone F, Roberti G, Micera A, Busanello A, Bonini S, Quaranta L, Agnifili L and Manni G: Exploring serum levels of brain derived neurotrophic factor and nerve growth factor across glaucoma stages. PLoS One 12: e0168565, 2017.

4. Mesentier-Louro LA, De Nicolò S, Rosso P, De Vitis LA, Castoldi V, Leocani L, Mendez-Otero R, Santiago MF, Tirassa P, Rama $\mathrm{P}$, et al: Time-dependent nerve growth factor signaling changes in the rat retina during optic nerve crush-induced degeneration of retinal ganglion cells. Int J Mol Sci 18: E98, 2017.

5. Hussein F, Antonescu C and Karshafian R: Ultrasound and microbubble induced release from intracellular compartments. BMC Biotechnol 17: 45, 2017.

6. Luo H, Huang WX, Yang C, Zhao JQ, Liu S, Xu YS and Liu CW: Therapeutic efficacy and mechanism of action of ginsenoside Rg1 in treating acute hepatic failure in mice. Zhonghua Gan Zang Bing Za Zhi 25: 217-222, 2017 (In Chinese).

7. Garcia TB, Hollborn M and Bringmann A: Expression and signaling of NGF in the healthy and injured retina. Cytokine Growth Factor Rev 34: 43-57, 2017.

8. Kimura A, Namekata K, Guo X, Harada C and Harada T: Neuroprotection, growth factors and BDNF-TrkB signalling in retinal degeneration. Int J Mol Sci 17: E1584, 2016.

9. Sun ZG, Chen LP, Wang FW, Xu CY and Geng M: Protective effects of ginsenoside Rg1 against hydrogen peroxide-induced injury in human neuroblastoma cells. Neural Regen Res 11: $1159-1164,2016$
10. Huang L, Liu LF, Liu J, Dou L, Wang GY, Liu XQ and Yuan QL: Ginsenoside Rg1 protects against neurodegeneration by inducing neurite outgrowth in cultured hippocampal neurons. Neural Regen Res 11: 319-325, 2016.

11. Huo DS, Zhang M, Cai ZP, Dong CX, Wang H and Yang ZJ: The role of nerve growth factor in ginsenoside Rg1-induced regeneration of injured rat sciatic nerve. J Toxicol Environ Health A 78: 1328-1337, 2015.

12. Wang B, He L, Cui B and Lv H: Protection of ginsenoside Rg1 on central nerve cell damage and the influence on neuron apoptosis. Pak J Pharm Sci 27 (Suppl 6): 2035-2040, 2014.

13. Li YB, Wang Y, Tang JP, Chen D and Wang SL: Neuroprotective effects of ginsenoside Rg1-induced neural stem cell transplantation on hypoxic-ischemic encephalopathy. Neural Regen Res 10: 753-759, 2015.

14. GuoX,GuoS,PanL,RuanL,GuY andLaiJ: Anti-microRNA-21/221 and microRNA-199a transfected by ultrasound microbubbles induces the apoptosis of human hepatoma HepG2 cells. Oncol Lett 13: 3669-3675, 2017.

15. Luo J, Zhou X, Diao L and Wang Z: Experimental research on wild-type p53 plasmid transfected into retinoblastoma cells and tissues using an ultrasound microbubble intensifier. J Int Med Res 38: 1005-1015, 2010.

16. Rokicki W, Zalejska-Fiolka J, Pojda-Wilczek D, Hampel A, Majewski W, Ogultekin S and Mrukwa-Kominek E: Differences in serum oxidative status between glaucomatous and nonglaucomatous cataract patients. BMC Ophthalmol 17: 13, 2017.

17. Panchal SS, Patidar RK, Jha AB, Allam AA, Ajarem J and Butani SB: Anti-inflammatory and antioxidative stress effects of oryzanol in glaucomatous rabbits. J Ophthalmol 2017: 1468716, 2017.

18. Mumcu UY, Kocer I, Ates O and Alp HH: Decreased paraoxonase1 activity and increased malondialdehyde and oxidative DNA damage levels in primary open angle glaucoma. Int J Ophthalmol 9: 1518-1520, 2016.

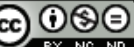

This work is licensed under a Creative Commons Attribution-NonCommercial-NoDerivatives 4.0 International (CC BY-NC-ND 4.0) License. 